\section{Multi-parametric single cell evaluation defines distinct drug responses in healthy hematologic cells that are retained in corresponding malignant cell types}

Muntasir M. Majumder, ${ }^{1}$ Aino-Maija Leppä, ${ }^{1}$ Monica Hellesøy, ${ }^{2}$ Paul Dowling, ${ }^{3}$ Alina Malyutina, ${ }^{1}$ Reidun Kopperud, ${ }^{4}$ Despina Bazou, ${ }^{5}$ Emma Andersson, ${ }^{6}$ Alun Parsons, ${ }^{1}$ Jing Tang, ${ }^{1}$ Olli Kallioniemi, ${ }^{1,7}$ Satu Mustjoki, ${ }^{6,8}$ Peter O'Gorman, ${ }^{5}$ Krister Wennerberg, ${ }^{1,9}$ Kimmo Porkka, ${ }^{8,10}$ Bjørn T. Gjertsen ${ }^{2,4}$ and Caroline A. Heckman $^{1}$

${ }^{1}$ Institute for Molecular Medicine Finland FIMM, Helsinki Institute of Life Science, University of Helsinki, Helsinki, Finland; ${ }^{2}$ Hematology Section, Department of Internal Medicine, Haukeland University Hospital, Bergen, Norway; ${ }^{3}$ Department of Biology, National University of Ireland, Maynooth, Ireland; ${ }^{4}$ Centre for Cancer Biomarkers CCBIO, Department of Clinical Science, University of Bergen, Bergen, Norway; ${ }^{5}$ Department of Hematology, Mater Misericordiae University Hospital, Dublin, Ireland; ' ${ }^{6}$ Department of Clinical Chemistry and Hematology, University of Helsinki, Finland; 'S Science for Life Laboratory, Department of Oncology and Pathology, Karolinska Institute, Solna, Sweden; ${ }^{8}$ Hematology Research Unit Helsinki, University of Helsinki, Helsinki, Finland; ${ }^{9}$ BRIC-Biotech Research and Innovation Centre, University of Copenhagen, Copenhagen, Denmark and ${ }^{10}$ Department of Hematology, Helsinki University Hospital Comprehensive Cancer Center, Helsinki, Finland

\section{ABSTRACT}

$\mathrm{I}$ nnate drug sensitivity in healthy cells aids identification of lineage specific anti-cancer therapies and reveals off-target effects. To characterize the diversity in drug responses in the major hematopoietic cell types, we simultaneously assessed their sensitivity to 71 small molecules utilizing a multi-parametric flow cytometry assay and mapped their proteomic and basal signaling profiles. Unsupervised hierarchical clustering identified distinct drug responses in healthy cell subsets based on their cellular lineage. Compared to other cell types, CD19+/B and CD56 $6^{+} / \mathrm{NK}$ cells were more sensitive to dexamethasone, venetoclax and midostaurin, while monocytes were more sensitive to trametinib. Venetoclax exhibited dose-dependent cell selectivity that inversely correlated to STAT3 phosphorylation. Lineage specific effect of midostaurin was similarly detected in CD19+/B cells from healthy, acute myeloid leukemia and chronic lymphocytic leukemia samples. Comparison of drug responses in healthy and neoplastic cells showed that healthy cell responses are predictive of the corresponding malignant cell response. Taken together, understanding drug sensitivity in the healthy cell-of-origin provides opportunities to obtain a new level of therapy precision and avoid off-target toxicity.

\section{Introduction}

During hematopoiesis, multipotent stem cells and pluripotent precursors undergo a complex differentiation program to generate a diverse set of blood cell types with wide-ranging phenotypes and functions. ${ }^{1}$ This process is initiated and driven by distinct signaling pathways linked to the different cellular lineages. ${ }^{2}$ It is likely that malignant hematopoietic cells exploit many of the signaling pathways essential for maintaining survival and specific functions of normal cells. Identification and understanding of normal hematopoietic cell type specific pathways could, therefore, be leveraged therapeutically as anti-cancer strategies against their malignant counterparts. For example, targeting B-cell antigen receptor (BCR) signaling with ibrutinib or idelalisib has proven highly effective in treating chronic lymphocytic leukemia (CLL). ${ }^{3,4}$ Conversely, modulating molecular targets shared between malignant and
Ferrata Storti Foundation

\section{Correspondence:}

CAROLINEA. HECKMAN

caroline.heckman@helsinki.fi

MUNTASIR MAMUN MAJUMDER

muntasir.mamun@helsinki.fi

Received: January 24, 2019

Accepted: August 22, 2019.

Pre-published: August 22, 2019.

doi:10.3324/haematol.2019.217414

Check the online version for the most updated information on this article, online supplements, and information on authorship \& disclosures: www.haematologica.org/content/105/6/1527

\section{(C)2020 Ferrata Storti Foundation}

Material published in Haematologica is covered by copyright. All rights are reserved to the Ferrata Storti Foundation. Use of published material is allowed under the following terms and conditions:

https://creativecommons.org/licenses/by-nc/4.0/legalcode. Copies of published material are allowed for personal or internal use. Sharing published material for non-commercial purposes is subject to the following conditions:

https://creativecommons. org//icenses/by-nc/4.0/legalcode, sect. 3. Reproducing and sharing published material for commercial purposes is not allowed without permission in writing from the publisher. 
healthy cells may give rise to untoward effects related to these entities. Although seminal studies have contributed to the understanding of signaling diversities across blood cells, ${ }^{5-8}$ a detailed characterization of cell-type specific vulnerabilities within the hematopoietic hierarchy is still lacking.

Cell-based phenotypic screens of primary cells have shown tremendous potential to identify novel therapeutics in leukemia and to explore novel indications for approved drugs. ${ }^{9,10}$ However, classical drug screening methods that assess the sum of all cellular effects in the bone marrow (BM) or blood restrict the ability to evaluate drug responses in populations affected by rare diseases and is influenced by the more abundant cell types in the sample. Flow cytometry presents a functional platform for dissecting the complexity of hematopoiesis, allowing characterization of the different cell populations. Applying flow cytometry in functional screens allows for a higher throughput (HTS) assessment of vulnerabilities to a large set of oncology drugs in leukemic cells with improved precision, and to compartmentalize drug responses between malignant and healthy cell subsets. However, preclinical flow cytometric-based high throughput functional screens are still limited by numerous washing steps and small cell population numbers, which can compromise the robustness of the assay.

In this study, we developed a high throughput no-wash flow cytometry assay that enabled us to monitor dose responses of 71 oncology compounds simultaneously on multiple hematopoietic cell populations defined by their surface antigen expression. To map the drug responses to the proteome and basal signaling profiles of the different cell types, we utilized mass spectrometry (MS) and mass cytometry (CyTOF) in both healthy and malignant hematologic samples. Finally, we compared inhibition profiles for those small molecules in a cohort of 281 primary samples representing a diverse set of hematologic malignancies to assess whether healthy cell-specific responses can be exploited in a leukemic context. A graphical overview of the study and cohorts is provided in Figure 1. Our results strongly suggest that drug responses are highly specific to cell lineages and often linked to intrinsic cell signaling present in those cell types. We provide evidence that cell-specific responses could potentially be applied to identify new clinical applications of therapies and discover relevant nononcogenic-dependent activities of small molecules.

\section{Methods}

\section{Patient specimens and cohorts}

Bone marrow and peripheral blood (PB) samples from 332 donors were collected after written informed consent (Studies: 239/13/03/00/2010, 303/13/03/01/2011, REK2016/253 and REK2012/2247) following protocols approved by local institutional review boards (Helsinki University Hospital Comprehensive Cancer Center and Haukeland University Hospital) in compliance with the Declaration of Helsinki. Samples were allocated to four patient cohorts (I-IV). Cohort I included three healthy PB samples used for flow cytometry screening with 71 drugs, plus three acute myeloid leukemia (AML) and ten multiple myeloma (MM) samples which were tested with bortezomib, clofarabine, dexamethasone, omipalisib, venetoclax and navitoclax. Cohort II included 17 samples from two healthy, eight AML with $(n=5)$ or without FLT3-ITD mutations $(\mathrm{n}=3)$, and seven CLL patients tested against midostaurin, trametinib and dasatinib. Cohort III $(n=281)$ included $231 \mathrm{BM}$ aspirates from a diverse collection of leukemia and 50 MM patients (CD138+ enriched). Four healthy BM aspirates subjected to magnetic bead-based enrichment using EasySep ${ }^{\text {TM }}$ human CD138, CD3, CD19, CD14 and CD34 positive selection kits (StemCell Technologies), served as healthy cell-of-origin samples for comparison against the malignant cell counterparts. CyTOF was performed on 14 samples in Cohort IV. PB from healthy donors ( $n=3)$, AML ( $n=6)$, B-cell acute lymphoblastic leukemia (BALL) ( $n=2)$, and matched BM samples from the same healthy donors were included. An overview of the cohorts and experimental design is provided in Figure 1.

\section{Proteome analysis}

$10 \mu \mathrm{g}$ of whole cell protein lysates, prepared from purified CD3, CD19 and CD14 fractions from healthy (n=2) and MM (n=4) samples, were digested and loaded (500 ng) on to a Q-Exactive mass spectrometer connected to a Dionex Ultimate 3000 (RSLCnano) chromatography system (Thermo Scientific). Protein identification and label-free quantification (LFQ) normalization of tandem mass spectrometry (MS/MS) data were performed using MaxQuant v1.5.2.8.

\section{Mass cytometry}

For mass cytometry (CyTOF), the 14 samples described in cohort IV were fixed, barcoded (Fluidigm), pooled into a single sample and stained with the antibody panels (Online Supplementary Table S1). Acquisition of samples was performed using a Helios mass cytometer (Fluidigm). Data were analyzed using FlowJo v.10.2 and Cytobank (Cytobank Inc.).

\section{High throughput flow cytometry and cell viability assay}

High throughput flow cytometry (HTFC) assays were performed in both 384-well ( $\mathrm{n}=3,71$ drugs, 5 concentrations) and 96well plate formats $(n=33)$ using IntelliCyt iQue Screener PLUS. A detailed optimization protocol is provided in the Online Supplementary Methods. A list of the antibodies is provided in Online Supplementary Table S1. Data were analyzed using ForeCyt software (Intellicyt). The gating strategy, cell composition and list of compounds are provided in Online Supplementary Figures S1-S3. CellTiter-Glo ${ }^{\oplus}$ luminescent viability assay was used based on a previously described method..$^{9,16}$

\section{Statistical analysis of drug sensitivity data}

Cell counts (HTFC) or luminescence intensity were used as input for Dotmatics (Dotmatics Ltd.) or Graphpad Prism 8.0 to generate dose response graphs, which were subsequently applied to calculate drug sensitivity score (DSS) as described by Yadav et al. ${ }^{16}$ Comparisons between groups were tested with ANOVA and with Tukey's multiple comparison test to derive significance. A two-tailed $P<0.05$ was considered significant.

\section{Results}

\section{Distinct drug response profiles in hematologic cell subsets are tied to cell lineages}

To simultaneously monitor drug effects on a large collection $(n=71)$ of samples in multiple cell types, we applied a multiplexed, no-wash flow cytometry-based assay (detailed in the Online Supplementary Methods). We first tested ex vivo response to the 71 compounds (Online Supplementary Table S2 and Online Supplementary Figure S3) in B (CD19+), natural killer (NK, CD56+), T-helper cells $\left(\mathrm{THC}, \mathrm{CDB}^{+} \mathrm{CD}^{+}\right.$), cytotoxic T lymphocytes (CTL, 
$\mathrm{CD}^{+} \mathrm{CD}^{+}$), NK-T cells (NK-T, $\mathrm{CD}^{+} \mathrm{CD}^{+} 6^{+}$), and monocytes $\left(\mathrm{CD} 14^{+}\right)$using three healthy blood samples to generate a global view of response profiles. Unsupervised hierarchical clustering of DSS of the screened samples segregated in three major clusters based on cellular lineages (Figure 2A). Monocytes formed a single cluster and displayed selective sensitivity to MEK/ERK inhibitors and the kinase inhibitor dasatinib (Figure 2A). The MEK inhibitor trametinib was similarly active in BM derived $\mathrm{CD} 14^{+}$cells from healthy and AML samples (Online Supplementary Figure S4A). However, reduced efficacy of dasatinib in BM monocytes was noted compared to those derived from blood (Online Supplementary Figure S4B). B and NK cells showed similar drug response profiles with higher sensitivity to the glucocorticoid dexamethasone, BCL2 inhibitor venetoclax and pan-kinase inhibitor midostaurin

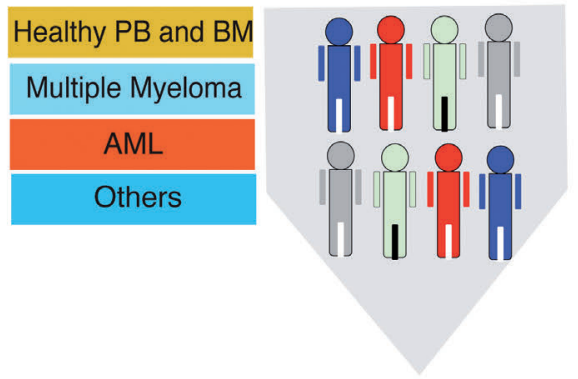

Identify cell lineage specific drug responses in healthy and patient samples

\section{Multiplexed Flow Cytometry}
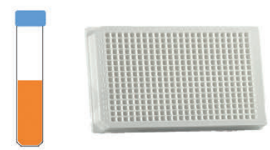

384 well format 71 drugs 6 cell subtypes 3 healthy samples

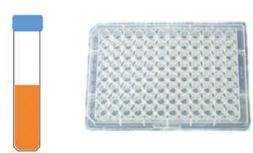

96 well format 6 drugs 10 cell subtypes

3 healthy, $3 \mathrm{AML}$ and $10 \mathrm{MM}$ samples
Protein expression profiles of monocytes, $T$ and $B$ cells Mass Spectometry

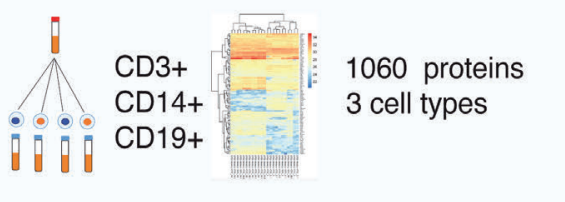

Identify drug responses in cell-of-origin that are retained in malignancies

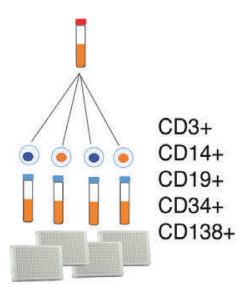

Drug response in enriched $\mathrm{CD} 3$, CD14, CD19, CD34 and CD138 cells from 5 healthy BM samples

\section{Cell Viability Assay}

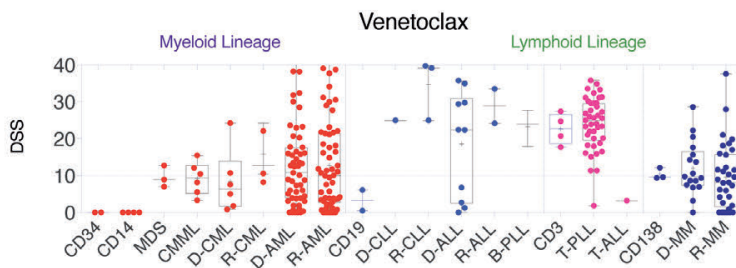

Comparison of drug responses in healthy cell of origin to 281 patient samples derived from $A M L, C M L, C M M L$, $\mathrm{PLL}, \mathrm{ALL}, \mathrm{CLL}, \mathrm{MM}$ and other hematological malignancies
Figure 1. Overview of the study. Schematic diagram summarizing the study design, datasets and analytical framework of the study. Bone marrow (BM) and peripheral blood (PB) samples from both healthy individuals and cancer patients were subjected to drug sensitivity assessment. Single cell drug sensitivity assay using the iQue ${ }^{\circledR}$ Screener PLUS flow cytometer was performed in 96and 384-well plates to monitor drug effects on ten and six hematopoietic cell subtypes, respectively. Immunophenotypic details and cellular proportions of the analyzed cell types are provided in Online Supplementary Figure $S 1 A-D$, Figure $S 2$ and Online Supplementary Table S3, respectively. 71 drugs in 384-well plates and six drugs in 96-well plates were tested. Proteomic analysis was performed on three cell subsets (monocytes, $T$ and $B$ cells) from two healthy individuals and four myeloma patients. Basal phosphorylation of nine signaling proteins involved in MAPK, JAKSTAT, PI3K-AKT-mTOR and NF-KB signaling was monitored in 14 samples. Healthy BM samples from four healthy individuals were subjected to CD34, CD3, CD14, CD19 and CD138 cell enrichment and tested against 71 small molecules with cell viability as the end point readout using the CellTiter$\mathrm{Glo}^{\circledast}$ assay. A comparison of ex vivo drug response in healthy and corresponding malignant cell types was performed for six drugs in 281 primary patient samples representing different hematologic malignancies. Samples included both published and unpublished datasets from chronic myeloid leukemia (CML, $\mathrm{n}=13),{ }^{11,12}$, chronic myelomonocytic leukemia (CMML, $\mathrm{n}=11),{ }^{12}$ myelodysplastic syndromes (MDS, $n=4$ ), acute myeloid leukemia (AML, $\mathrm{n}=145$ ), 9,12 B-cel acute lymphoblastic leukemia (BALL, $n=14),{ }^{13}$ chronic lymphocytic leukemia ( $C L L, n=4),{ }^{12}$ T-cell prolymphocytic leukemia (T-PLL, $\mathrm{n}=40$ ), ${ }^{14}$ multiple myeloma (MM $\mathrm{n}=50),{ }^{15}$ and other hematologic malignancies $(n=6)$. PLL: prolymphocytic leukemia. 
compared to other cell types. Except for NK-T cells from one donor, all T-cell subsets formed a distinct cluster.

Based on observations from the primary screen, we focused our in-depth analysis on six compounds displaying either non-selective (proteasome inhibitor bortezomib and nucleoside analog clofarabine) or cell-selective (dexamethasone, venetoclax, pan-BCL2 inhibitor navitoclax and $\mathrm{PI} 3 \mathrm{~K} / \mathrm{mTOR}$ inhibitor omipalisib) responses. Sixteen samples (Cohort I) derived from $10 \mathrm{MM}, 3 \mathrm{AML}$ and 3 healthy donors were tested utilizing two antibody panels

A

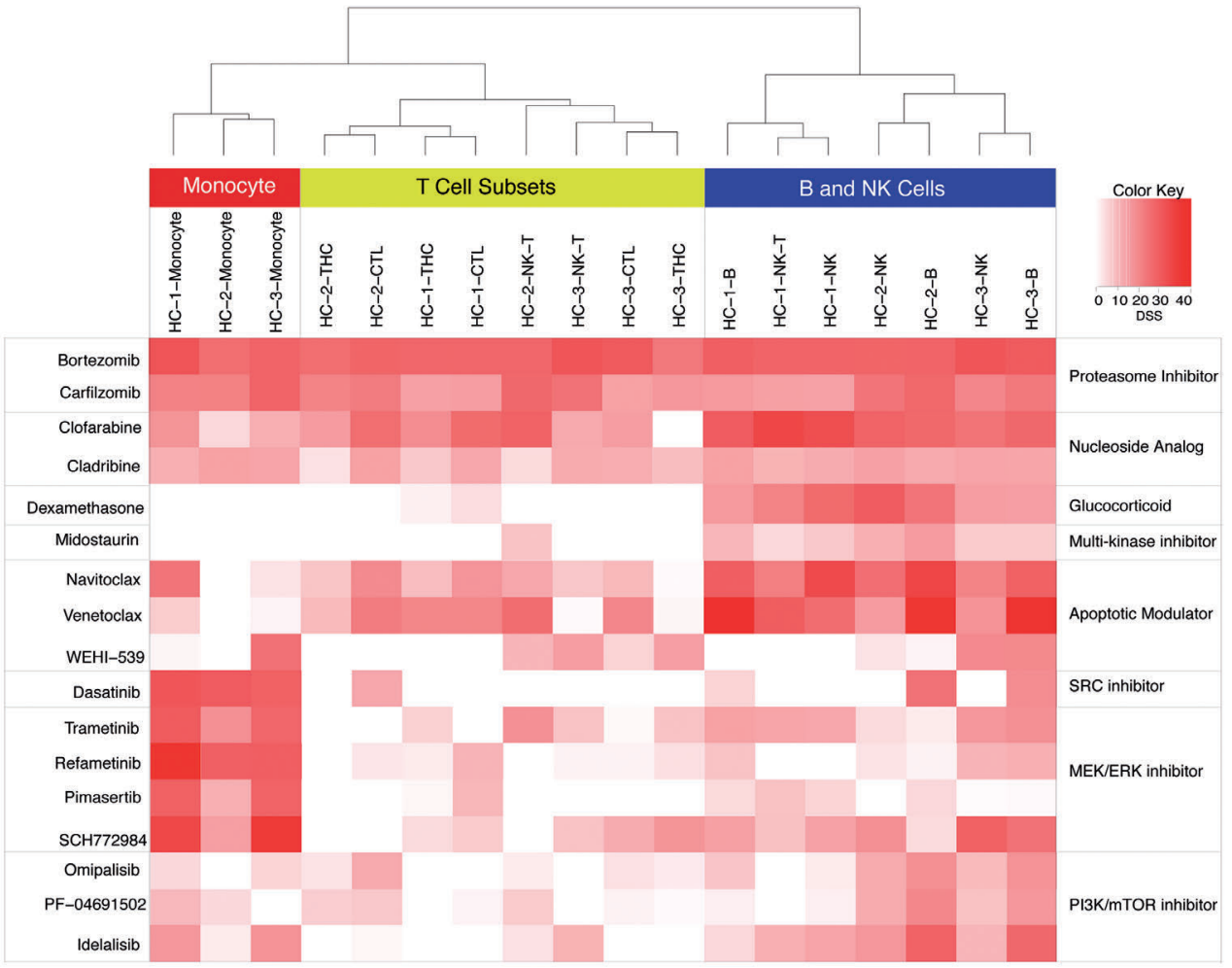

- $\mathrm{CD} 34+\mathrm{CD} 38-/ \mathrm{HSC}$ - CD34+CD38+/CPC - CD14+/Monocyte - CD45+SSC++/Granulocyte a CD19+/B

a CD138+/Plasma Cell $\approx$ CD56+/NK $=\mathrm{CD3}+\mathrm{CD} 56+/ \mathrm{NK}-\mathrm{T}=\mathrm{CD} 3+\mathrm{CD} 4+/$ Helper T $\approx \mathrm{CD} 3+\mathrm{CD} 4-/$ Cytotoxic T Lymphocyte

B

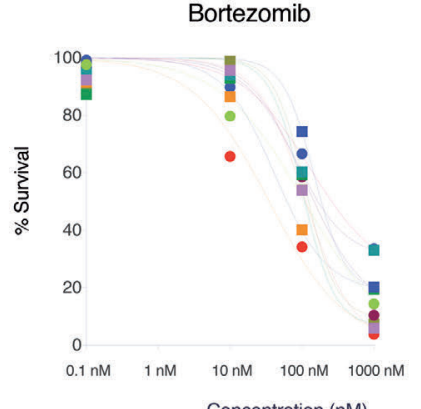

Omipalisib

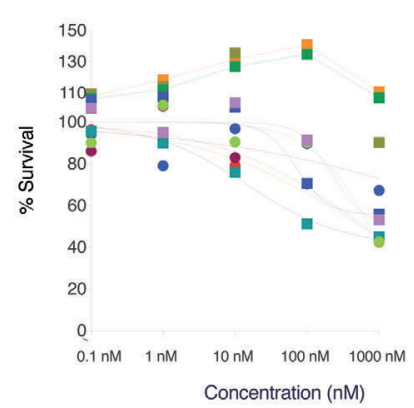

Clofarabine

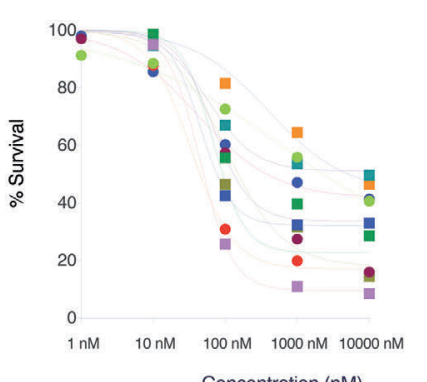

Concentration (nM)

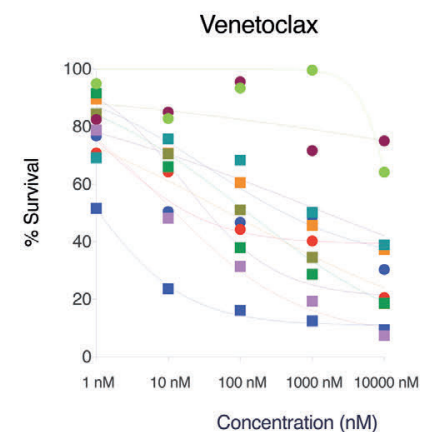

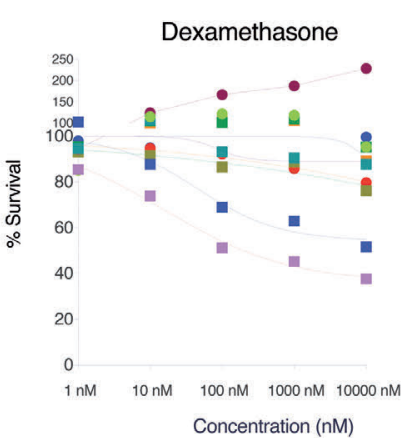

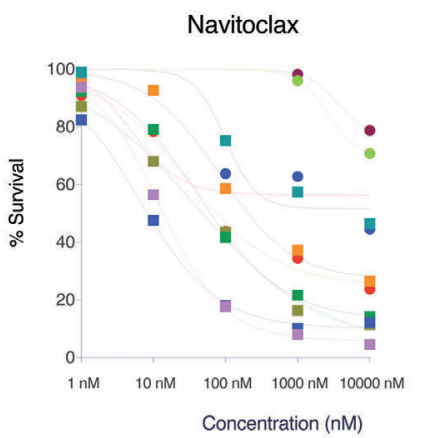

Figure 2. Distinct drug responses observed in immune subsets are tied to cell lineages. (A) A drug sensitivity score (DSS), ${ }^{16}$ which is a modified form of the area under the curve (AUC) calculation, was used to quantitate drug responses among the different detected cell populations. Higher DSS values (DSS $>10$ ) indicate higher sensitivity to the individual drug. The heatmap displays a summary view of hierarchical clustering analysis with DSS scores for six cell subsets in three peripheral blood (PB) samples from healthy controls (marked as HC-1, 2 and 3). Immunophenotyping of hematopoietic cell types was performed on the basis of their known surface antigen expression profiles (see also Online Supplementary Figure S1A). Monocytes and T-cell subsets formed two separate clusters. B and natural killer (NK) cells had similar drug response patterns. Small molecules that were tested along with their functiona classes are displayed in Online Supplementary Figure S3. DSS scores and IC50 values for all 71 drugs are provided in Online Supplementary Table S2. (B) Differential effect of bortezomib, dexamethasone, clofarabine, venetoclax, navitoclax and omipalisib on hematopoiet ic cell subsets presented as mean values from 16 samples derived from three healthy, three acute myeloid leukemia (AML) and ten multiple myeloma (MM) patients (Cohort I) Highest variation was observed for BCL2 inhibitors (venetoclax and navitoclax) and dexamethasone between myeloid and lymphoid lineages. Monocytes were resistant to both of these drug classes. A concentrationdependent increase in numbers of $\mathrm{CD}^{+}$cells was observed for omipalisib at 10 and 100 $\mathrm{nM}$. The proportion of cells detected in these analyzed samples are presented in Online Supplementary Table S3. 
(Online Supplementary Table S1) in 96-well plates to provide a direct comparison between cell types derived from healthy donors and those derived from patients with identical immunophenotypes. Moreover, this enabled detection of drug responses in rare cell subsets, such as plasma cells $\left(\mathrm{CD} 138^{+}\right)$and progenitor cells $\left(\mathrm{CD} 34^{+} \mathrm{CD} 38\right.$ or $\left.\mathrm{CD} 34^{+} \mathrm{CD} 38^{+}\right)$.

While ex vivo response to the proteasome inhibitor bortezomib was detected in most cell types (Figure $2 \mathrm{~B}$ ), CD138 ${ }^{+} \mathrm{CD} 38$ plasma cells were resistant compared to $\mathrm{CD} 138^{+} \mathrm{CD} 38^{+}$or other cells (Online Supplementary Figure S5). A higher response to the nucleoside analog clofarabine was noted for $\mathrm{CD}^{+} \mathrm{CD} 4$ and $\mathrm{CD} 34^{+} \mathrm{CD} 38^{+}$cells compared to $\mathrm{CD}^{+} \mathrm{CD} 4^{+}$or $\mathrm{CD} 34^{+} \mathrm{CD} 38$ cells. Dexamethasone depleted $\mathrm{CD} 9^{+}$and $\mathrm{CD}^{+} 6^{+}$cells and induced a dose-dependent increase in the $\mathrm{CD}_{14}^{+}$cell count (Figure 2B). T-cell subsets were insensitive to $\mathrm{PI} 3 \mathrm{~K} / \mathrm{mTOR}$ inhibitor omipalisib. A similar effect for several molecules targeting the PI3K-mTOR signaling axis was observed in $\mathrm{CD}^{+}$enriched cells tested with a cell viability assay (Online Supplementary Figure S6). Surprisingly, an increase in $\mathrm{CD}^{+}$cell count was noted at concentrations of 10 and $100 \mathrm{nM}$ (Figure 2B). Apart from individual variations, distinct drug efficacies associated with healthy cell lineages were detected equally in all patient specimens (Figure 2B and Online Supplementary Figure S7).

\section{Venetoclax shows variable dose-dependent efficacy on hematopoietic cell types}

Preclinical and clinical activity of venetoclax has been well documented for several B-cell malignancies. ${ }^{17-20} \mathrm{We}$ measured the response to venetoclax, which is highly selective for BCL2, and navitoclax, which targets BCL2, BCL-W and BCL-XL. Both inhibitors were similarly effective against lymphocytes (Figure 2B). Within the lymphocyte compartment, the highest sensitivity to venetoclax was detected for $\mathrm{CD}_{19}{ }^{+}$cells (Figures $2 \mathrm{~B}$, and $3 \mathrm{~A}$ and $\mathrm{B}$ ) with the majority of samples (Cohort I) responding at subnanomolar concentrations (IC50, 0.4-12 nM). Activity towards $\mathrm{CD}^{+} \mathrm{CD} 4$ cells was observed at 10-100-fold higher concentrations (IC50, 8-140 nM). A further reduction in response (Figure $3 \mathrm{~B}$ ) was observed for $\mathrm{CD}^{+} 6^{+}$, $\mathrm{CD}^{+} \mathrm{CD}^{+}$and $\mathrm{CD}^{+} \mathrm{CD}^{+} 6^{+}$cells (IC50, $\cong 100 \mathrm{nM}$ to 1 $\mu \mathrm{M})$. Monocytes and granulocytes were sensitive to BCL2 inhibitors only at the highest concentration $(10 \mu \mathrm{M})$ and were considered largely resistant (Figure 2B). This dosedependent effect on cell types is particularly relevant when treating elderly patients, with frequent age-related

A

Venetoclax
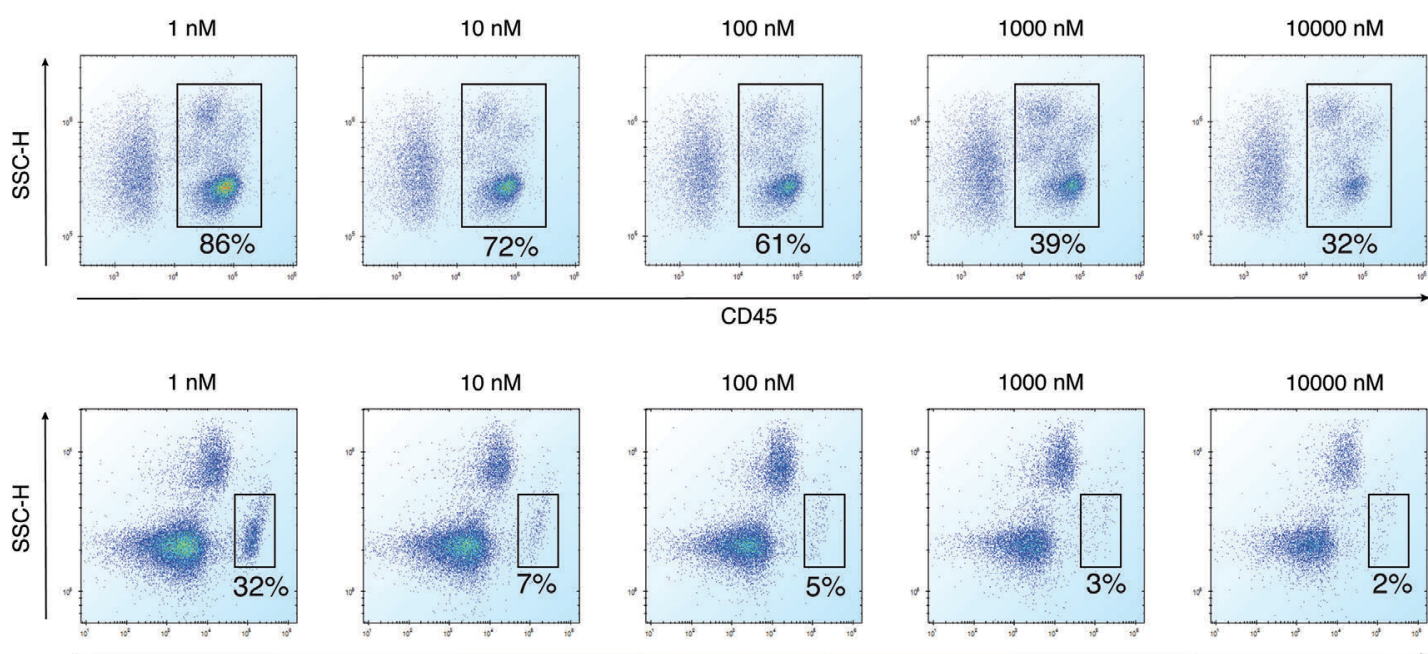

CD19

B

Venetoclax

CD19+

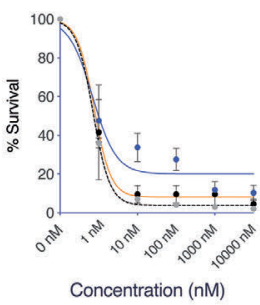

CD56+

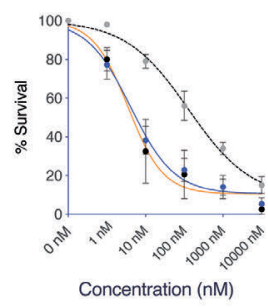

$\mathrm{CD} 3+\mathrm{CD} 4+$

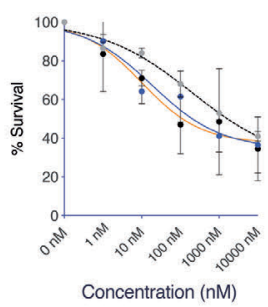

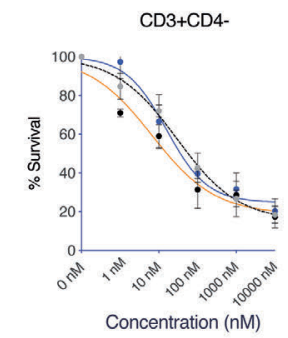

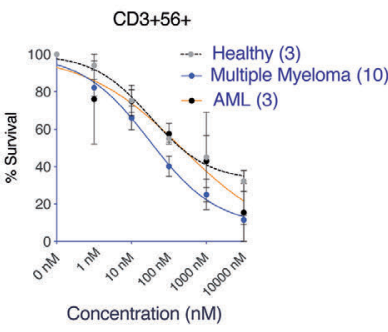

Figure 3. Variable dose-dependent activity of venetoclax on leukocytes. (A) Scatter diagram displaying dose-dependent cytotoxicity of venetoclax (1-10,000 nM) in $\mathrm{CD} 45^{+}$(upper panel) and $\mathrm{CD} 45^{+} \mathrm{CD} 19^{+}$(lower panel) cells for a single patient. (B) Averaged dose response graphs generated for different immune cell subtypes derived from healthy $(n=3)$, acute myeloid leukemia $(A M L)(n=3)$, and multiple myeloma $(M M)(n=10)$ samples showed venetoclax sensitivity in $C D 19+/ B$ cells with an $\mathrm{IC}_{50}<1 \mathrm{nM}$. CD3 ${ }^{+} \mathrm{CD} 4$ cytotoxic T cells were more sensitive compared to $\mathrm{CD}^{+} \mathrm{CD} 4^{+} \mathrm{T}$-helper cells. Data are presented as mean \pm standard error of mean responses for the tested samples in each disease group. Mean $I_{50}$ values for the analyzed samples are listed in Online Supplementary Table S4. 
decline in drug metabolism and excretion, ${ }^{21,22}$ which can result in drug accumulation leading to unintended effects on other immune cells. Venetoclax displayed similar cellspecific effects in all tested samples, whether healthy or malignant, suggesting the variation in response is purely lineage specific.

\section{Lineage specific effect of midostaurin on CD19+ cells is comparable to FLT3-mutated acute myeloid leukemia cells}

In our primary screen, we observed selective depletion of $B$ and NK cells in $P B$ samples $(n=3)$ treated with midostaurin (Figure 2A and Online Supplementary Figure S8A), which is approved for treating FLT3-mutated AML and systemic mastocytosis. ${ }^{23,24}$ To evaluate $\mathrm{CD}^{2} 9^{+}$cell specificity in malignant cells such as in CLL and to compare the response to FLT3-ITD-mutated AML cells, we tested midostaurin in 17 additional samples (Cohort II) derived from healthy $(\mathrm{n}=2)$, CLL ( $\mathrm{n}=7)$, and AML patients with wild-type (WT) FLT3 $(\mathrm{n}=3)$ or harboring the FLT3ITD mutation $(\mathrm{n}=5)$. Variable sensitivity was noted in the CD $34{ }^{+} \mathrm{CD} 38$ population, presumably leukemic stem cells, from all tested AML samples regardless of FLT3 mutation status (Figure 4A). CD34+CD38+/blast cells from all FLT3ITD mutated AML samples were sensitive (median $\mathrm{IC}_{50}$, $554 \mathrm{nM}$ ) (Figure 4B). Remarkable sensitivity ( $\mathrm{IC}_{50}, 16 \mathrm{nM}$ ) was detected in the $\mathrm{CD} 34^{+} \mathrm{CD} 38^{+}$fraction from one of the three WT samples (Online Supplementary Figure S8B). While CD34 $\mathrm{CD} 38$ cells from healthy donors were insensitive, $\mathrm{CD} 34^{+} \mathrm{CD} 38^{+}$cells from one healthy individual responded similarly to FLT3-ITD-mutated AML samples (Online Supplementary Figure S8B). Importantly, we observed high efficacy against $\mathrm{CD} 19^{+}$cells in all tested samples including those derived from CLL patients (Figure 4C, D and Online Supplementary Figure S8C) indicating a lineage specific effect. The effect on $\mathrm{CD} 19^{+} / \mathrm{B}$ cells (median IC $_{50}, 314 \mathrm{nM}$ ) was comparable to FLT3-ITD mutated AML CD34+CD38 (blast) cells (Figure $4 \mathrm{~B}$ and C). Our results suggest a need for further investigation to evaluate midostaurin efficacy in diseases affecting B-cell lineages such as CLL.

\section{Characterizing protein abundance and basal cell signaling contributing to innate cellular response to therapies}

Having determined lineage specificity of the tested small molecules, we next explored whether protein abundance or basal signaling profiles of specific cell populations could explain the innate cellular responses. We also investigated whether healthy cells share identical basal activity for signaling proteins as patient-derived cells or whether basal intracellular signaling was deregulated during malignant transformation. To characterize and compare the proteomic background of healthy hematopoietic
A

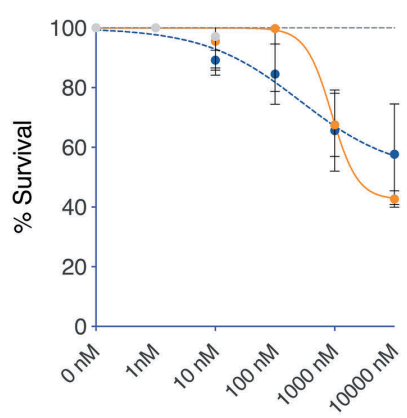

Concentration (nM)

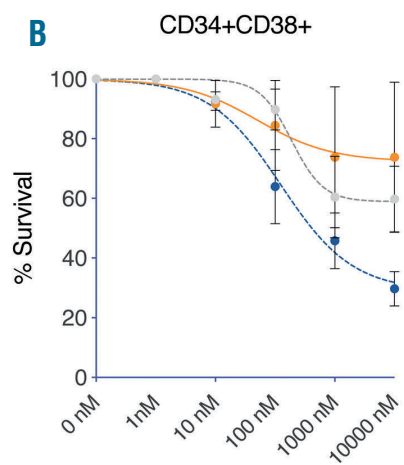

Concentration (nM)

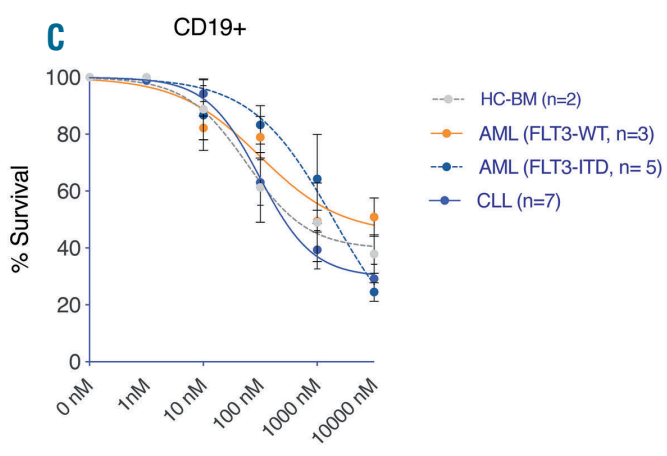

Concentration (nM)
D

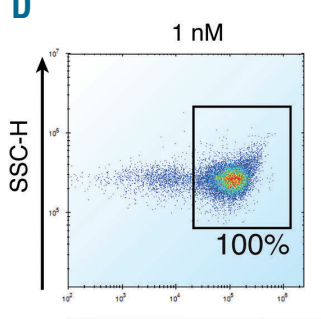

$10 \mathrm{nM}$

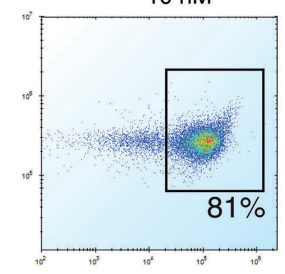

$100 \mathrm{nM}$

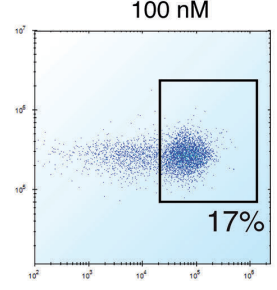

$1000 \mathrm{nM}$

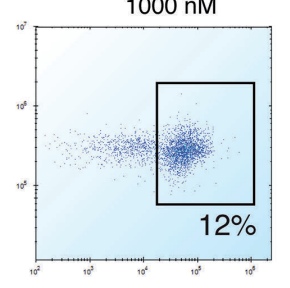

$10000 \mathrm{nM}$

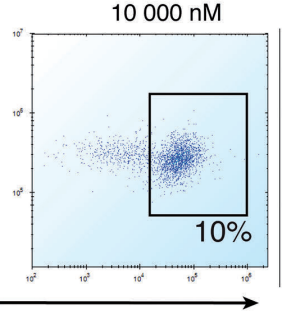

CD19

Figure 4. Effect of midostaurin on the viability of $\mathrm{CD} 34^{+} \mathrm{CD} 38, \mathrm{CD} 34^{+} \mathrm{CD} 38^{+}$and $\mathrm{CD} 19^{+}$cells derived from healthy donors, and acute myeloid leukemia (AML) or chronic lymphocytic leukemia (CLL) patients. Averaged dose response curves for disease categories are presented as mean \pm standard error of mean. (A) While midostaurin treatment had no effect on $\mathrm{CD} 34^{+} \mathrm{CD} 38$ cells from healthy individuals, variable sensitivity was detected in $\mathrm{AML}$ samples. $(B)$ CD $34^{+} \mathrm{CD} 38^{+}$cells derived from FLT3-ITD-mutated AML samples displayed similar sensitivity (median $\mathrm{IC}_{50}, 554 \mathrm{nM}$ ). (C) CD19+ cells derived from healthy donor or patient samples showed comparable sensitivity at a median $\mathrm{IC}_{50}$ of $319 \mathrm{nM}$. Individual dose response curves are provided in Online Supplementary Figure S8C. (D) Scatter plot showing dose responses for midostaurin in CD19+ cells from a CLL patient. The percentage of CD19+ live cells present in midostaurin-treated wells compared to untreated cells is displayed numerically on the plot. Cellular proportions for these samples are provided in Online Supplementary Table S5. Related IC ${ }_{50}$ values are provided in Online Supplementary Table S6. 
A

CD14, IL1B, p38 MAPK, CTSS, M6PR,

IGLL1, IGHA1, CORO1B, HSPB1,GSR

ATP6VOD1, ATG3, LAMP2, RAC1, IGF2R

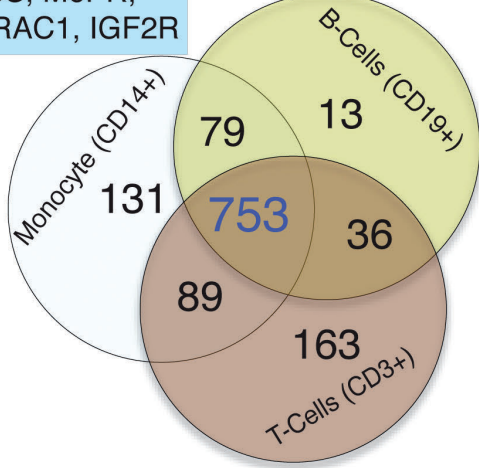

GZMA, PRF1, CD8A, CD247, ZAP70, LCK, GNAS

PPP1C, ATP5AI, NDUFA8, NDUFB3, UQCRB

B
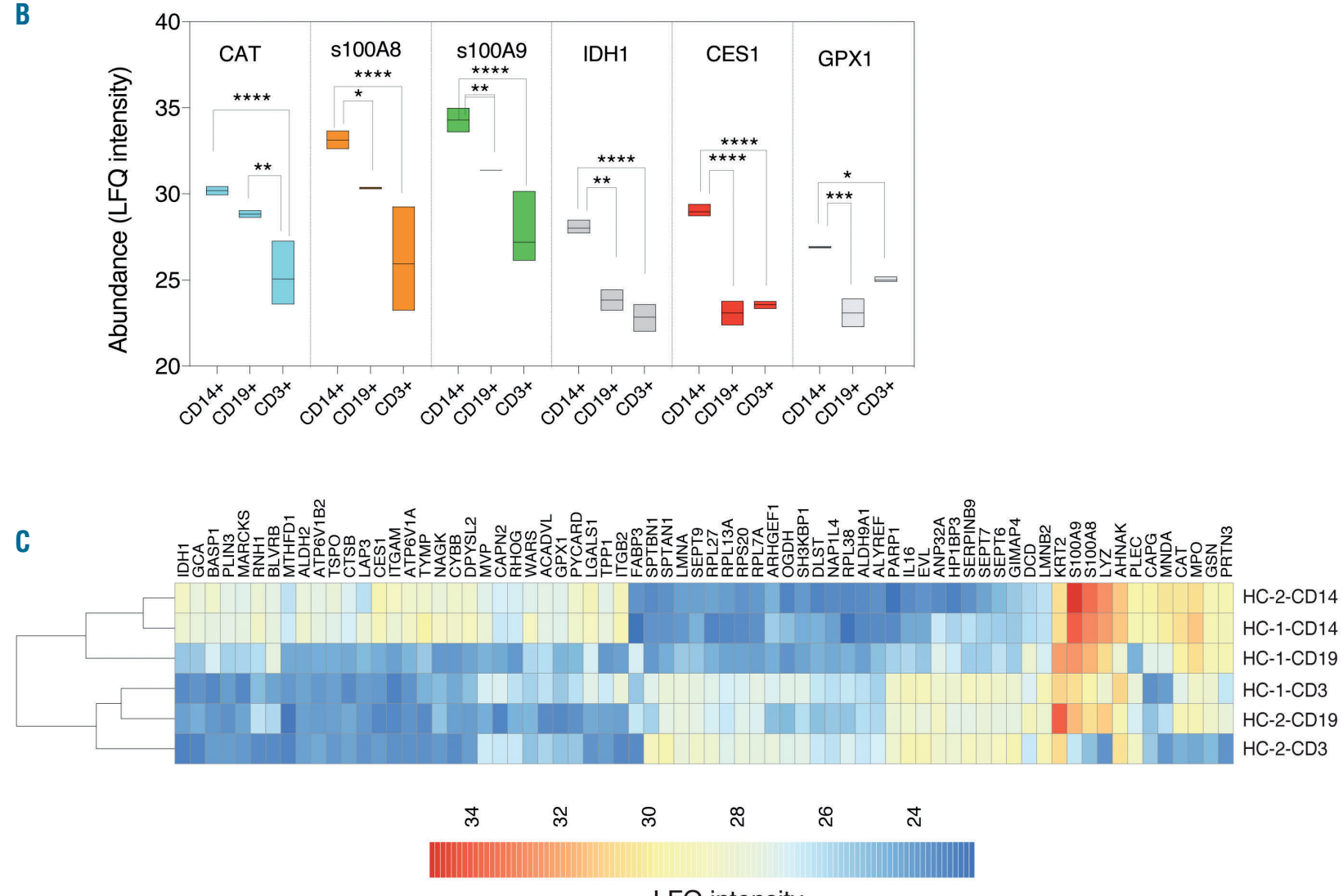

LFQ intensity

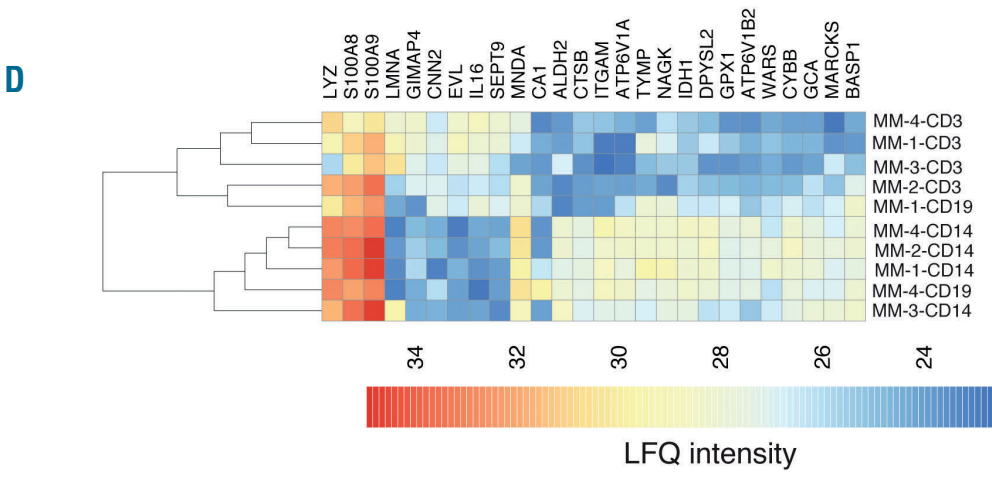

Figure 5. Differences in protein expression between hematopoietic cell subsets. (A) Venn diagram representation of unique and commonly detected proteins using mass spectrometry across healthy $T$ cells, B cells and monocytes. Uniquely detected proteins corresponded to their cellular functions shown in the inset boxes. Out of 1,060 proteins detected in lysates from two healthy and four multiple myeloma (MM) samples, abundance of 753 commonly detected proteins was compared between the three cell types. A complete list of proteins and their corresponding abundance values are provided in Online Supplementary Table S7. (B) Significantly higher expression of S100A8/A9, CAT, IDH1, CES1 and GPX1 was detected in healthy monocytes. (C and D) Heatmap summarizing the expression of proteins that significantly discriminated three cell types in healthy control $(\mathrm{HC})$ and MM samples, respectively (false discovery rate $<0.05$ ). The data presented here are normalized label-free quantification (LFQ) intensity values for the proteins. 
cells, we utilized a mass spectrometry-based quantitative proteomics approach to profile B cells $\left(\mathrm{CD} 19^{+}\right)$, T cells $\left(\mathrm{CD}^{+}\right)$and monocytes $\left(\mathrm{CD} 14^{+}\right)$derived from two healthy donors (Figure 5). We then employed CyTOF to compare the basal activity of nine proteins (in healthy and leukemic cell subsets) involved in MAPK, JAK-STAT, NF-кB and PI3K-mTOR signaling, which are commonly activated in many hematologic malignancies ${ }^{25-28}$ (Figure 6). By sample barcoding and subsequent pooling prior to antibody staining, CyTOF allows for direct comparison of the phosphorylation level of target proteins between multiple donors with high fidelity. ${ }^{19,20}$

Monocytes show higher expression of calprotectin (S100A8/S100A9), which is associated with dexamethasone resistance - By quantitative mass spectrometry-based proteomics, a total of 1,060 proteins were detected. Among these, 163, 131 and 13 proteins were only identified in CD3, CD14 and CD19 lysates, respectively (Figure 5A, Online Supplementary Table S7). The uniquely expressed proteins were associated with biological processes consistent with the functional differences between these cell types (Online Supplementary Figure S9). For instance, the proteome signature in monocytes was enriched in biological processes related to phagosome maturation (ATP6VD1, CTSS, M6PR), autophagy (ATG3, LAMP2), PPAR- $\alpha /$ RAR $\alpha$ activation (IL1 $\beta$, p38 MAPK, GPD2, PLCG2), and STAT3 signaling (IGF2R, RAC1, p38 MAPK). Immunoglobulins (IGLL1, IGHA1) were identified in Bcell fractions. T cells expressed proteins related to T-cell receptor signaling (CD8A, CD247, LCK, ZAP70), granzyme signaling (GZMA, PRF1), and oxidative phosphorylation (ATP5I, NDUFA8, NDUFB3, UQCRB). Besides observed differences in the abundance of proteins, variable expression in commonly detected proteins was noted (Figure $5 \mathrm{~B}$ and $\mathrm{C}$ ). Enzymes associated with scavenging reactive oxygen species such as catalase (CAT) and glutathione peroxidase 1 (GPX1) were expressed at a significantly higher $(P<0.001)$ level in monocytes (Figure $5 \mathrm{~B})$. In addition, monocytes exhibited elevated expression of isocitrate dehydrogenase 1 (IDH1), carboxylesterase 1 (CES1), and inflammatory protein calprotectin, a heterodimer of two proteins S100A8/S100A9 that can mediate dexamethasone resistance in patients. ${ }^{29,30}$ We further compared the protein expression profiles for these cell subsets between healthy and four MM patients, and found an identical pattern of expression for CAT, GPX1 and S100A8/9 proteins (Figure 5D). While expression of 16 proteins differed between healthy and MM samples [false discovery rate $(\mathrm{FDR})<0.05]$, no significant differences were noted for $\mathrm{CD}^{+} 4^{+}$and $\mathrm{CD}^{+}$lysates ( $<3$ proteins).

Mapping shared signaling activities in healthy and leukemic hematopoietic cell subsets - NF- $\kappa B$ phosphorylation was detected in most cell types. Compared to other cell types, higher $\mathrm{pNF}-\kappa \mathrm{B}$ was detected in T/CD3 cells (Online Supplementary Figure S10). Significantly higher mTOR signaling, as measured by p4E-BP1 and pPLC- $\gamma 1$, was observed in healthy $\mathrm{CD} 34^{+} \mathrm{CD} 38^{+}$cells, monocytes, granulocytes (neutrophils) and B cells (Figure 6A-C). These cell types also tended to have elevated sensitivity to omipalis$\mathrm{ib}$ (PI3K/mTOR inhibitor) compared to other cell types in healthy or malignant samples (Figure $2 \mathrm{~B}$ and Figure $7 \mathrm{E}$ ). $\mathrm{T}$ cells lacking sensitivity to PI3K/mTOR inhibitors showed reduced mTOR signaling activity (Figure 6A-C).
CD $34^{+} \mathrm{CD} 38^{+}$cells also exhibited high levels of ERK phosphorylation (Figure 6A-C). ERK phosphorylation status, however, did not correlate to increased trametinib sensitivity in monocytes. An inverse relation between pSTAT3 levels and venetoclax sensitivity was observed among the different cell populations. Heightened levels of pSTAT3 were detected in monocytes and granulocytes, which lacked sensitivity to venetoclax (Figure 6A-D). In contrast, a lower level of pSTAT3 was observed in venetoclax sensitive B and NK cells. Two related but distinct cell types, $\mathrm{CD}^{+} \mathrm{CD}^{+}$and $\mathrm{CD}^{+}{ }^{+} \mathrm{CD}^{+}{ }^{+} \mathrm{T}$ cells, exhibited a difference in the level of pSTAT3 (Figure 6B) that might explain their subtle difference in sensitivity to venetoclax (Figure 6D). Comparison of signaling patterns detected in healthy $\mathrm{PB}$ or BM cells to corresponding leukemic cells expressing identical surface markers revealed remarkable similarity (Figure 6A-C), strengthening their association with cellular phenotypes. Furthermore, monitoring changes in signaling pattern for these proteins upon treatment with increasing concentrations $(0 \mathrm{nM}, 10 \mathrm{nM}$ and $10 \mu \mathrm{M})$ of venetoclax in healthy $\mathrm{PB}(\mathrm{n}=3)$, revealed that the directionality or magnitude of signaling changes for some of these proteins (i.e. pPLC- $\gamma 1$ and pCREB) were also similar across these cell types (Online Supplementary Figure S11).

\section{Innate drug sensitivities in cell subsets are retained in their malignant counterparts in different hematologic malignancies}

To further confirm the similarity in drug responses between healthy and patient-derived cell subsets observed using the single cell assay, we compared ex vivo drug responses detected in bead-enriched healthy cells $\left(\mathrm{CD}^{+}, \mathrm{CD}^{4} 4^{+}, \mathrm{CD} 19^{+}, \mathrm{CD}^{4} 4^{+}\right.$and $\left.\mathrm{CD} 138^{+}\right)$to a cohort of 281 primary samples derived from multiple hematologic malignancies. For these analyses, we generated data using the CellTiter-Glo ${ }^{\circledR}$ viability assay. In agreement with non-selective effects detected on healthy cell types, bortezomib activity was detected in a wide range of hematologic malignancies (Figure 7A). The highest clofarabine efficacy was observed in $\mathrm{CD}^{+} \mathrm{T}$ cells and in the T-cell prolymphocytic leukemia (T-PLL) patient subset (Figure 7B), which is reflective of the clinical success observed with other purine analogs (fludarabine or cladribine) in T-PLL. Reduced activity of the purine analog clofarabine was detected in both healthy and myeloma derived CD138+ cells. Although dexamethasone was found to be most effective in B-ALL and CLL, modest ex vivo effects were observed in other lymphocytic and plasma cell malignancies, including T-ALL, T-PLL, B-PLL, and MM (Figure 7C). Disease-specific acquisition of sensitivity was also noted in a subset of AML patients, which was undetectable in healthy $\mathrm{CD}^{+} 4^{+}$(Figure 7C) or $\mathrm{CD}^{+} 4^{+} \mathrm{CD}_{38}{ }^{+}$(Figure 4B) cells. T-cell malignancies, similar to healthy $\mathrm{T}$ cells, showed no response to omipalisib. Consistent with responses observed in healthy B cells, a higher response to venetoclax was detected in malignant B-cell types (Figure 7F). T-PLL samples also exhibited sensitivity to venetoclax, which has been tested more recently in two T-PLL patients with measurable clinical benefit. $^{32}$ Venetoclax response agreed with navitoclax responses in B-cell diseases (Figure 7F). Increased sensitivity to navitoclax compared to venetoclax was detected in CML, T-ALL, and MM samples (Figure 7E and F). Bcell specific responses to midostaurin were detected in CLL and ALL samples (Figure 4C and Online 
Supplementary Figure S12). Collectively, the comparison of lineage specific drug responses between healthy individuals and those derived from malignancies where the cell-of-origin is affected shows remarkable similarity.
These results also highlight the fact that innate drug responses are often retained during cellular transformation, which could guide identification of lineage specific anticancer therapies for leukemia.

A

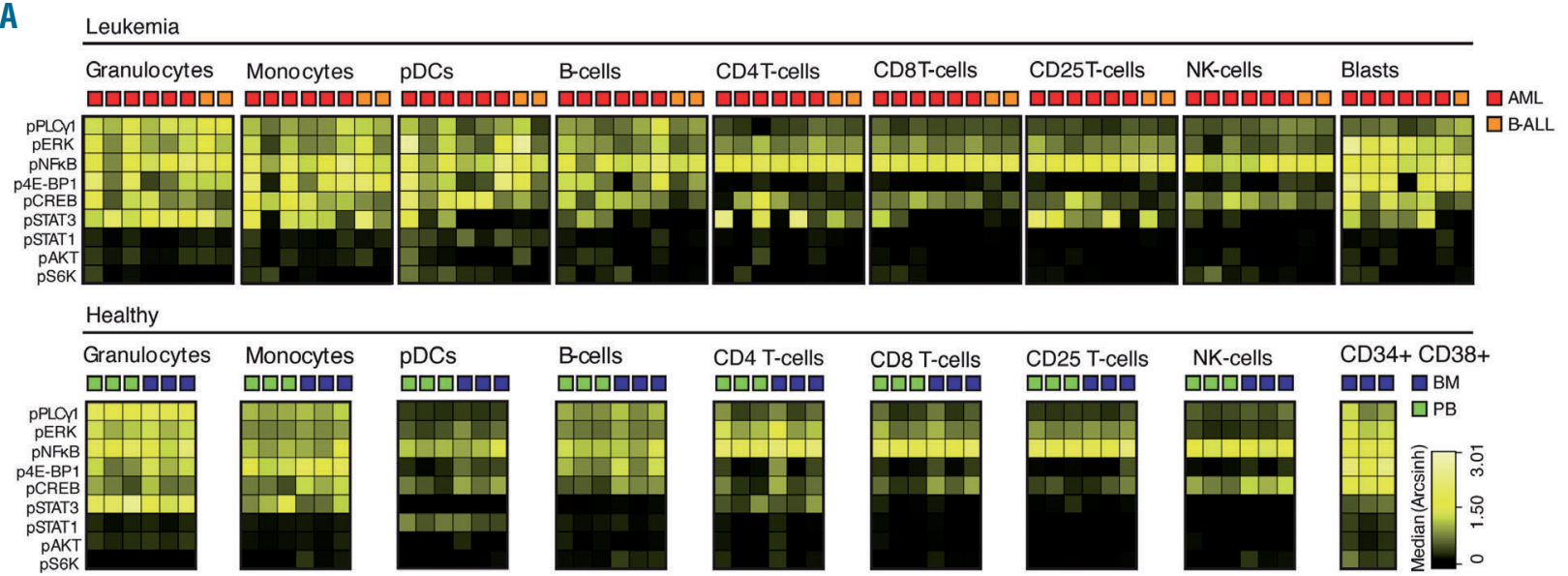

B

Healthy

Leukemia
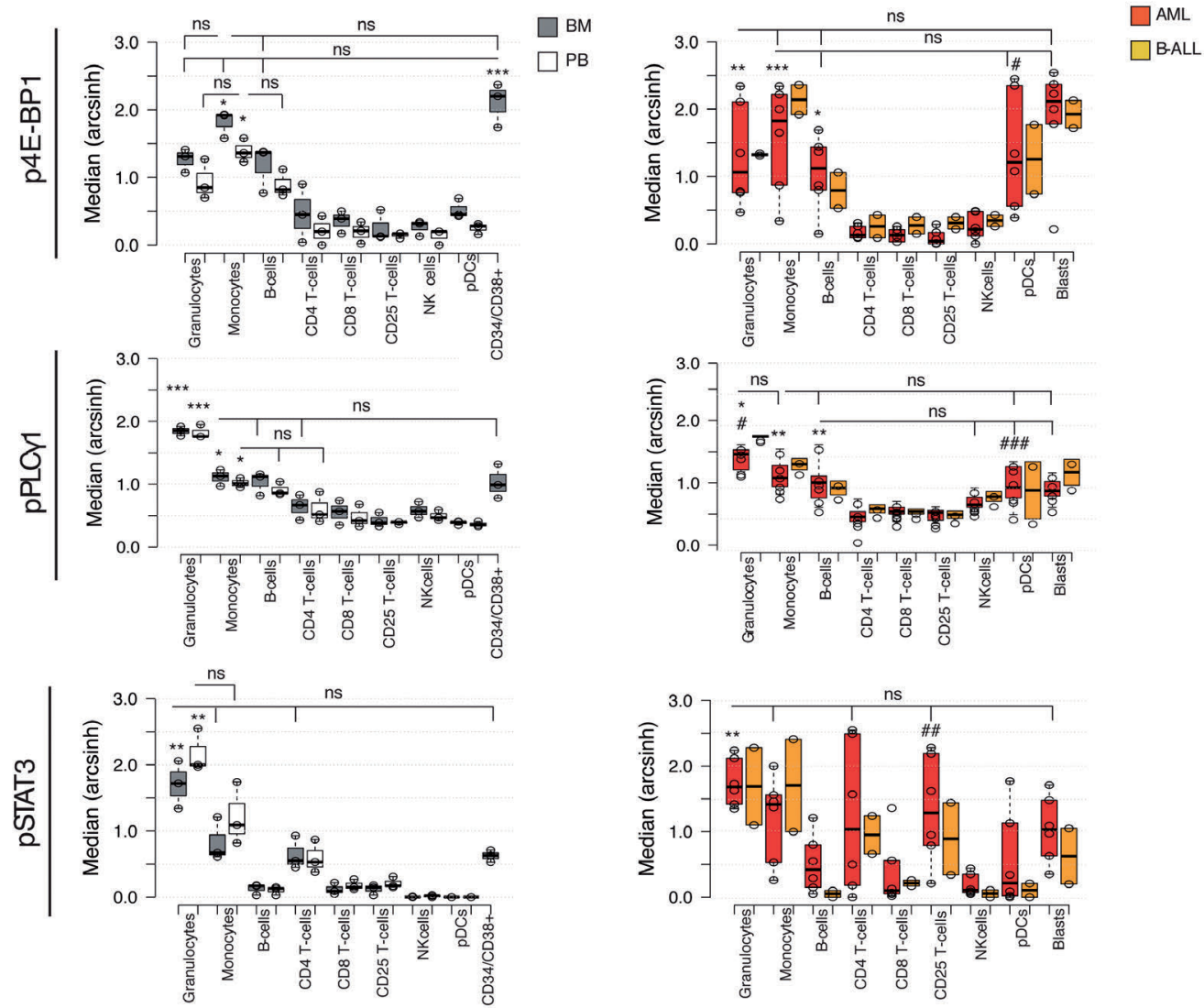

Figure 6. Mass cytometry (CyTOF) profiling of basal signaling patterns in nine healthy cell subsets. (A) Summary of the basal phosphorylation state of 9 signaling proteins associated with NF-KB, PI3K-AKT-mTOR (AKT, 4E-BP1, PLCG1 and p70-S6K), JAK-STAT (STAT1 and STAT3), MAPK (ERK and CREB) in three healthy individuals. Matched peripheral blood (PB) and bone marrow $(\mathrm{BM})$ from the same healthy donors were profiled. Phosphorylation profiles for the indexed phosphoproteins were investigated in corresponding cell subsets in leukemic samples: acute myeloid leukemia (AML) and B-cell acute lymphoblastic leukemia (B-ALL). (B) Box plot representation of population medians of p4EBP1, pPLC 1 and pSTAT in healthy PB and BM (left) and leukemic samples (right). Center lines of boxes show medians; box limits indicate the $25^{\text {th }}$ and $75^{\text {th }}$ percentiles as determined by R software; whiskers extend 1.5 times the interquartile range from the $25^{\text {th }}$ and $75^{\text {th }}$ percentiles, outliers are represented by dots; data points are plotted as open circles. *Significant difference (two-way ANOVA, Tukey's HSD) between all corresponding populations, unless specified as not significant (ns). "Significance between AML and the corresponding healthy populations in both PB and BM. There were no significant differences between PB and BM in any population for each of the measured phosphoproteins, $* P<0.05, * * P<0.005, * * * P<0.0005$. Healthy PB and BM ( $n=3$ ), AML ( $n=6)$, B-ALL ( $n=2)$. (Continued on the next page) 
C

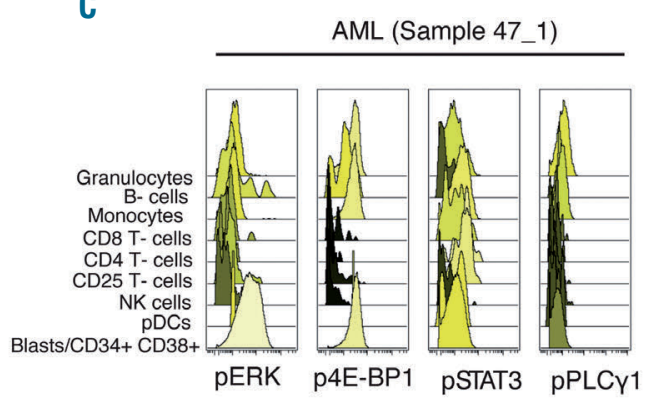

D

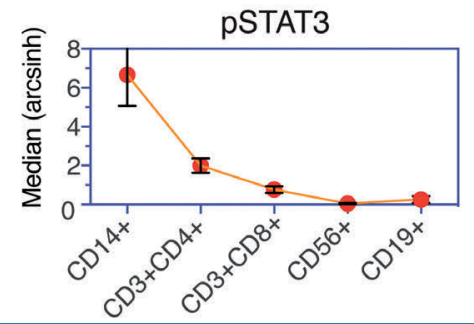

Healthy BM (FRI-113)

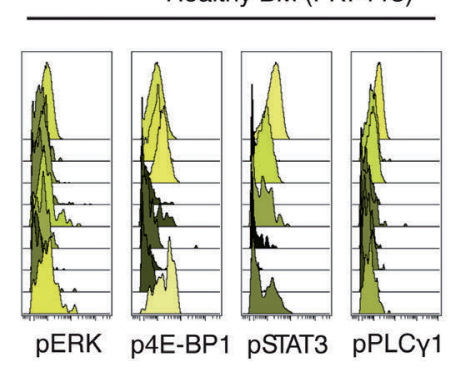

E

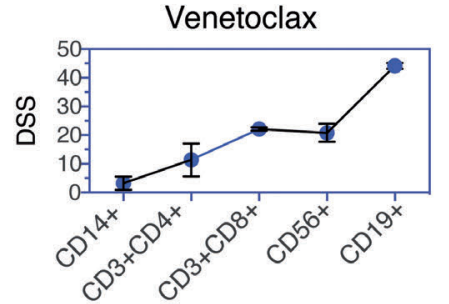

Healthy PB (FRI-113)

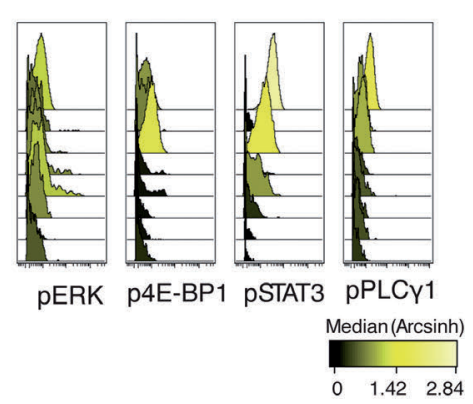

Figure 6. (continued from the previous page) (C) Stacked histogram representations of pERK, p4E-BP1, pSTAT3 and pPLC 1 phosphorylation status across cell types in an AML patient and healthy donor samples (paired BM and PB). Immunophenotype of the presented samples are provided in Online Supplementary Figure S1B. (D) Phosphorylation of STAT3 in five healthy cell types from three healthy individuals presented as mean \pm standard error of mean (SEM) arcsinh values derived from mass cytometry analysis. (E) Venetoclax response in cell types displayed as SEM of drug sensitivity score (DSS) values for three healthy donors. A higher response to venetoclax correlated with reduced phosphorylation of STAT3. PDC: plasmacytoid dendritic cells; NK: natural killer.

\section{Discussion}

Applying a high throughput, multi-parametric singlecell assay, we aimed to assess the diversity in drug effects on multiple cell populations in individual donor samples. Therapeutic efficacy was determined ex vivo using complex mixtures of cells to more realistically recapitulate the native environment. Our results demonstrate that cell subtypes are drastically different from each other with respect to macromolecule abundance, signaling profiles and drugresponse patterns against a diverse collection of anticancer drugs. As such, this study provides a comprehensive portrait of the drug sensitivity landscape in hematologic cell subsets and reveals drug responses that are tied to specific cell lineages. Importantly, cell subset-specific sensitivity and resistance mechanisms were clearly reflected in their malignant counterpart.

Variation in drug responses can arise in healthy hematologic cell subsets due to differences in signaling state and transcriptional programs attributed to their cellular function or phenotype. Many of the signaling events are tightly regulated in cellular subsets ${ }^{2}$ and may often determine their innate sensitivity to drug treatment. For example, the basal state of pSTAT3 could explain the diversity of the ex vivo responses to BCL2 inhibitors observed between healthy cell subsets. In relation to this, we have previously reported a critical role of the JAK-STAT pathway in venetoclax resistance in AML, which could be reversed using a combination of the JAK1/2 inhibitor ruxolitinib, and venetoclax. ${ }^{32}$ Although changes in signaling behaviors are commonly detected in malignant cells,? understanding basal signaling in the cell-of-origin is fundamental to identify cancer vulnerabilities or off target effects that are lineage specific.

Profiling healthy and malignant cells (from multiple hematologic malignancies) revealed that the cell-of-origin associated signaling events and drug responses were also retained in the disease context of the affected cell type. Hence, the profiling presented here provides new targeting opportunities in previously unexplored disease indications. Venetoclax sensitivity in B cells may reflect its efficacy in CLL and other B-cell malignancies ${ }^{33}$ which could also be exploited in diseases where B-cell depletion is considered, i.e. in rheumatoid arthritis. Similar to other studies, 34,35 we demonstrated midostaurin efficacy in CLL and ALL, which may be ascribed to the intrinsic response detected in $\mathrm{CD}_{19}{ }^{+}$cells. Efficacy was also detected in $\mathrm{CD} 34^{+} \mathrm{CD} 38^{+}$cells from AML ( $\left.\mathrm{n}=1\right)$ with WT FLT3. This observation may reflect its efficacy reported in AML with WT FLT3. ${ }^{24}$ Although NK-cell malignancies are rare, they are often highly aggressive and refractory in nature. ${ }^{36} \mathrm{We}$ found that dexamethasone and midostaurin targeted NK cells with similar efficacy as B cells, implicating a potential clinical utility of these drugs for NKcell malignancies.

Expression of antioxidant enzymes and calprotectin (S100A8/S100A9) is associated with drug resistance, including resistance to dexamethasone, which has been documented in both hematologic and solid tumors. ${ }^{29,37-40}$ We noted that healthy monocytes displaying elevated expression of these proteins are intrinsically resistant to dexamethasone. Dexamethasone induced apoptosis of lymphocytes is attributed to free radical generation, an effect that can be counteracted by antioxidant enzymes such as catalase, which may itself confer in monocytes resistance to steroids. ${ }^{30,41}$ Furthermore, the monocyte expansion observed with dexamethasone treatment could be explained by its ability to mimic IL1B activation of IL1R, ${ }^{42}$ which stimulates their proliferation. This phenomenon had previously been observed in murine 

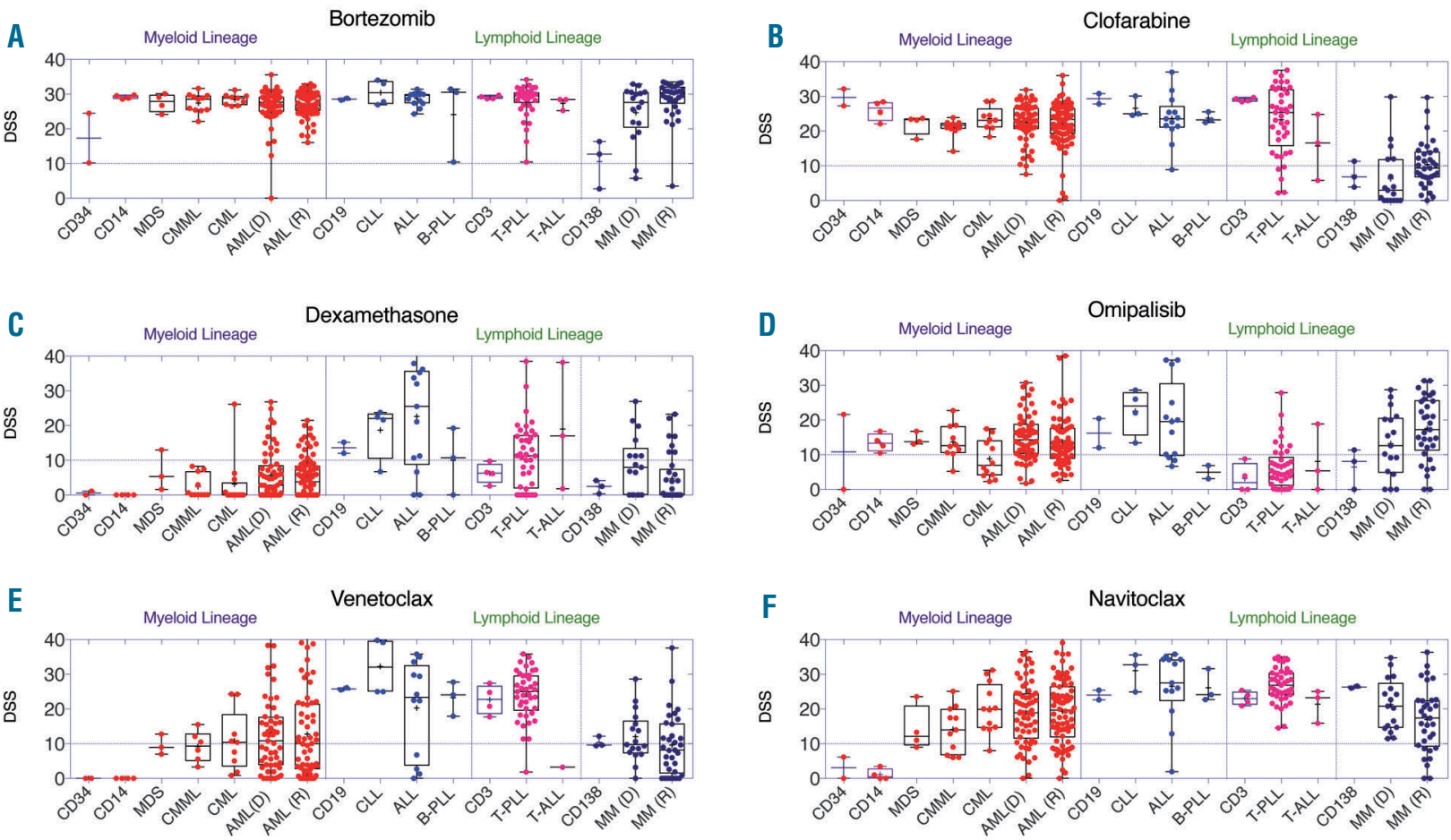

Figure 7. Systematic comparison of drug responses in healthy cell-of-origin and corresponding cell types from hematologic malignancies. (A-F) Ex vivo drug responses presented as drug sensitivity scores (DSS) of healthy cell types (CD3, $n=4 ; C D 14, n=4 ; C D 19, n=2 ; C D 34, n=2 ;$ and $C D 138$, $n=3$ ) were compared to malignant counterparts in a cohort of 281 primary samples for bortezomib, clofarabine, dexamethasone, omipalisib, venetoclax and navitoclax. Samples included both published and unpublished datasets generated at our facility for chronic myeloid leukemia $(C M L)(n=13),{ }^{10,11}$ chronic myelomonocytic leukemia $(C M M L)$ ( $\left.n=11\right),{ }^{12}$ myelodysplastic syndromes (MDS) $(n=4)$, acute myeloid leukemia (AML) $(n=145),{ }^{9,12}$ B-cell acute lymphoblastic leukemia (B-ALL) ( $\left.n=14\right),{ }^{13}$ chronic lymphocytic leukemia (CLL) $(n=4),{ }^{12}$ T-cell prolymphocytic leukemia (T-PLL) $(n=40),{ }^{14}$ multiple myeloma $(M M)(n=50)^{15}$ and other hematologic malignancies $(n=6)$. AML and MM samples were subdivided depending on whether they were derived from newly diagnosed (D) and relapsed (R) samples. T-PLL and MM samples were tested with enriched $\mathrm{CD}^{+}$and $\mathrm{CD} 138^{+}$cells. Results provide evidence that response in healthy cell subsets is predictive of responses observed in the malignant cell counterparts. A comparison between drug effects on $\mathrm{CD}_{14}{ }^{+}$and $\mathrm{CD} 34^{+}$cells derived from healthy individuals and AML samples are displayed in Online Supplementary Figure S13 and S14. B-PLL: B-cell prolymphocytic leukemia.

monocytes, ${ }^{43}$ as well in acute monocytic leukemia [AML, French-American-British (FAB) subtype M5] where glucocorticoid treatment may further lead to an increase in blast population, fueling disease progression. ${ }^{44}$ Therefore, understanding the cytoprotective mechanisms operating in healthy cell subsets could also provide crucial insights into drug resistance mechanisms in patients.

Cancer immunotherapies and drugs modulating the immune system are emerging as important treatment modalities for hematologic and solid tumors. ${ }^{45,46}$ Preserving cytotoxic lymphocytes is critical for their efficacy and may have significant consequences on long-lasting antitumor adaptive immunity, likely responsible for durable clinical responses. In our study, few inhibitors showed a selective effect on a single cell type; instead, effects were either directed to multiple cell types or in a non-selective manner to all exposed cells. For example, dexamethasone and midostaurin depleted $\mathrm{CD} 19^{+} \mathrm{B}$ cells and $\mathrm{CD} 56^{+} \mathrm{NK}$ cells. Similarly, venetoclax depleted $\mathrm{CD}^{+}{ }^{+} \mathrm{CD} 4$ cytotoxic $\mathrm{T}$ cells, among other cell types. Reduction of immune effector cells (mainly cytotoxic T cells and NK cells) are particularly relevant because of their key role in cancer immunosurveillance and immunotherapy. ${ }^{47}$ Therefore, profiling the unintended effects of small molecules on effector cells, which are used in combination with immunotherapies, is fundamental to the selection of rational combination partners, and to preserve the quality and quantity of immune cells in patients.

In summary, the findings presented in this study suggest that dissecting drug responses in hematologic cell lineages could represent an invaluable tool to reveal the full spectrum of cellular effects, identify novel drug resistance mechanisms, and to predict off target effects of small molecules. We envision that incorporating the assessment of cell lineage-specific drug responses into routine procedures in preclinical drug development holds great promise in identifying new therapeutic niches of small molecules and improve precision in therapies, particularly for hematologic malignancies.

\section{Acknowledgments}

The authors thank the patients, healthy donors and participating clinics for their generous contributions. Members of the High Throughput Biomedicine Unit at FIMM, Flow Cytometry Core Facility at UiB, and Mass Spectrometry Facility at Maynooth University, are greatly appreciated for their support and expertise. Special appreciation to Minna Suvela and Siv Knaappila for their excellent technical support. The Bergen Research Foundation is acknowledged for support of the Helios CyTOF instrumentation. $M H$ and BTG were supported by the Norwegian Cancer Society project n. 144345. The study was supported by funding from the Cancer Society of Finland. 


\section{References}

1. Jassinskaja M, Johansson E, Kristiansen TA, et al. Comprehensive Proteomic Characterization of Ontogenic Changes in Hematopoietic Stem and Progenitor Cells. Cell Rep. 2017;21(11):3285-3297.

2. Bendall SC, Simonds EF, Oiu P, et al. Singlecell mass cytometry of differential immune and drug responses across a human hematopoietic continuum. Science. 2011:332(6030):687-696.

3. Compagno $M$, Wang $Q$, Pighi $C$, et al. Phosphatidylinositol 3-kinase delta blockade increases genomic instability in B cells. Nature. 2017;542(7642):489-493

4. Robak T, Robak P. BCR signaling in chronic lymphocytic leukemia and related inhibitors currently in clinical studies. Int Rev Immunol. 2013;32(4):358-376.

5. Irish JM, Hovland R, Krutzik PO, et al. Single cell profiling of potentiated phospho-protein networks in cancer cells. Cell. 2004;118(2):217-228.

6. Bodenmiller B, Zunder ER, Finck R, et al. Multiplexed mass cytometry profiling of cellular states perturbed by small-molecule regulators. Nat Biotechnol. 2012;30(9):858-867.

7. Irish JM, Kotecha N, Nolan GP. Mapping normal and cancer cell signalling networks: towards single-cell proteomics. Nat Rev Cancer. 2006;6(2):146-155

8. Krutzik PO, Crane JM, Clutter MR, Nolan GP. High-content single-cell drug screening with phosphospecific flow cytometry. Nat Chem Biol. 2008;4(2):132-142.

9. Pemovska T, Kontro M, Yadav B, et al. Individualized Systems Medicine Strategy to Tailor Treatments for Patients with Chemorefractory Acute Myeloid Leukemia. Cancer Discov. 2013;3(12):1416-1429.

10. Pietarinen PO, Pemovska T, Kontro M, et al. Novel drug candidates for blast phase chronic myeloid leukemia from high-throughput drug sensitivity and resistance testing. Blood Cancer J. 2015;5:e309.

11. Pietarinen PO, Eide CA, Ayuda-Duran P, et al. Differentiation status of primary chronic myeloid leukemia cells affects sensitivity to BCR-ABL1 inhibitors. Oncotarget. 2017:8(14):22606-22615

12. Kontro M, Kumar A, Majumder MM, et al. HOX gene expression predicts response to BCL-2 inhibition in acute myeloid leukemia. Leukemia. 2017;31(2):301-309.

13. Eldfors S, Kuusanmaki H, Kontro M, et al. Idelalisib sensitivity and mechanisms of disease progression in relapsed TCF3-PBX1 acute lymphoblastic leukemia. Leukemia. 2017;31(1):51-57.

14. Andersson EI, Putzer S, Yadav B, et al. Discovery of novel drug sensitivities in TPLL by high-throughput ex vivo drug testing and mutation profiling. Leukemia. 2018;32(3):774-787.

15. Majumder MM, Silvennoinen R, Anttila P, et al. Identification of precision treatment strategies for relapsed/refractory multiple myeloma by functional drug sensitivity testing. Oncotarget. 2017;8(34):56338-56350.

16. Yadav B, Pemovska T, Szwajda A, et al. Quantitative scoring of differential drug sensitivity for individually optimized anticancer therapies. Sci Rep. 2014;4:5193

17. Cang S, Iragavarapu C, Savooji J, Song Y, Liu
D. ABT-199 (venetoclax) and BCL-2 inhibitors in clinical development. J Hematol Oncol. 2015;8:129.

18. Edelmann J, Gribben JG. Managing Patients with TP53-Deficient Chronic Lymphocytic Leukemia. J Oncol Pract. 2017;13(6):371 377.

19. Leonard JT, Rowley JS, Eide CA, et al. Targeting $\mathrm{BCL}-2$ and $\mathrm{ABL} / \mathrm{LYN}$ in Philadelphia chromosome-positive acute lymphoblastic leukemia. Sci Transl Med. 2016;8(354):354ra114.

20. Rosenthal A. Small Molecule Inhibitors in Chronic Lymphocytic Lymphoma and B Cell Non-Hodgkin Lymphoma. Curr Hematol Malig Rep. 2017;12(3):207-216.

21. Mangoni AA, Jackson SH. Age-related changes in pharmacokinetics and pharmacodynamics: basic principles and practical applications. $\mathrm{Br}$ J Clin Pharmacol. 2004;57(1):6-14.

22. EIDesoky ES. Pharmacokinetic-pharmacodynamic crisis in the elderly. Am J Ther 2007;14(5):488-498

23. Garcia JS, Percival ME. Midostaurin for the treatment of adult patients with newly diagnosed acute myeloid leukemia that is FLT3 mutation-positive. Drugs Today (Barc). 2017:53(10):531-543

24. Stone RM, Manley PW, Larson RA, Capdeville R. Midostaurin: its odyssey from discovery to approval for treating acute myeloid leukemia and advanced systemic mastocytosis. Blood Adv. 2018;2(4):444-453.

25. Kawauchi K, Ogasawara T, Yasuyama M Otsuka K, Yamada O. Regulation and importance of the PI3K/Akt/mTOR signaling pathway in hematologic malignancies. Anticancer Agents Med Chem. 2009;9(9):1024-1038

26. Yamada O, Kawauchi K. The role of the JAK-STAT pathway and related signal cascades in telomerase activation during the development of hematologic malignancies. JAKSTAT. 2013;2(4):e25256.

27. Ward AF, Braun BS, Shannon KM. Targeting oncogenic Ras signaling in hematologic malignancies. Blood. 2012;120(17):3397 3406.

28. Springuel L, Renauld JC, Knoops L. JAK kinase targeting in hematologic malignancies: a sinuous pathway from identification of genetic alterations towards clinical indications. Haematologica. 2015;100(10):12401253

29. Spijkers-Hagelstein JA, Schneider P, Hulleman E, et al. Elevated S100A8/S100A9 expression causes glucocorticoid resistance in MLLrearranged infant acute lymphoblastic leukemia. Leukemia. 2012;26(6):1255-1265.

30. Tome ME, Baker AF, Powis G, Payne CM, Briehl MM. Catalase-overexpressing thymocytes are resistant to glucocorticoid-induced apoptosis and exhibit increased net tumo growth. Cancer Res. 2001;61(6):2766-2773.

31. Boidol B, Kornauth C, van der Kouwe E, et al. First-in-human response of BCL-2 nhibitor venetoclax in T-cell prolymphocytic leukemia. Blood. 2017;130(23):2499-2503.

32. Karjalainen R, Pemovska T, Popa M, et al. JAK1/2 and BCL2 inhibitors synergize to counteract bone marrow stromal cellinduced protection of AML. Blood. 2017:130(6):789-802

33. Davids MS, Roberts AW, Seymour JF, et al. Phase I First-in-Human Study of Venetoclax in Patients With Relapsed or Refractory Non-Hodgkin Lymphoma. J Clin Oncol. 2017;35(8):826-833

34. Gallogly MM, Lazarus HM. Midostaurin: an emerging treatment for acute myeloid leukemia patients. J Blood Med. 2016;7:7383.

35. Ganeshaguru K, Wickremasinghe RG, Jones $\mathrm{DT}$, et al. Actions of the selective protein kinase $\mathrm{C}$ inhibitor PKC412 on B-chronic lymphocytic leukemia cells in vitro. Haematologica. 2002;87(2):167-176

36. Cheung MM, Chan JK, Wong KF. Natural killer cell neoplasms: a distinctive group of highly aggressive lymphomas/leukemias. Semin Hematol. 2003;40(3):221-232.

37. Reeb AN, Li W, Sewell W, et al. S100A8 is a novel therapeutic target for anaplastic thyroid carcinoma. I Clin Endocrinol Metab. 2015:100(2): E232-242.

38. Wang Y, Guo A, Liang X, et al. HRD1 sensitizes breast cancer cells to Tamoxifen by promoting S100A8 degradation. Oncotarget. 2017;8(14):23564-23574.

39. Yang M, Zeng P, Kang R, et al. S100A8 contributes to drug resistance by promoting autophagy in leukemia cells. PLoS One. 2014;9(5): e97242.

40. Yang XY, Zhang MY, Zhou Q, et al. High expression of S100A8 gene is associated with drug resistance to etoposide and poor prognosis in acute myeloid leukemia through influencing the apoptosis pathway. Onco Targets Ther. 2016;9:4887-4899.

41. Tome ME, Jaramillo MC, Briehl MM Hydrogen peroxide signaling is required for glucocorticoid-induced apoptosis in lymphoma cells. Free Radic Biol Med. 2011;51(11):2048-2059.

42. Dubois CM, Neta R, Keller JR, Jacobsen SE Oppenheim JJ, Ruscetti F. Hematopoietic growth factors and glucocorticoids synergize to mimic the effects of IL-1 on granulocyte differentiation and IL-1 receptor induction on bone marrow cells in vivo. Exp Hematol. 1993;21(2):303-310

43. Trottier MD, Newsted MM, King LE, Frake PJ. Natural glucocorticoids induce expansion of all developmental stages of murine bone marrow granulocytes without inhibiting function. Proc Natl Acad Sci U S A. 2008;105(6):2028-2033

44. Klein K, Haarman EG, de Haas V, Zwaan Ch M, Creutzig U, Kaspers GL. GlucocorticoidInduced Proliferation in Untreated Pediatric Acute Myeloid Leukemia Blasts. Pediatr Blood Cancer. 2016;63(8):1457-1460

45. Deligne C, Milcent B, Josseaume N, Teillaud JL, Siberil S. Impact of Depleting Therapeutic Monoclonal Antibodies on the Host Adaptive Immunity: A Bonus or a Maluse Front Immunol 2017:8:950.

46. Gandhi AK, Kang J, Havens CG, et al Immunomodulatory agents lenalidomide and pomalidomide co-stimulate $T$ cells by inducing degradation of $\mathrm{T}$ cell repressors Ikaros and Aiolos via modulation of the E3 ubiquitin ligase complex CRL4(CRBN.). Br Haematol. 2014;164(6):811-821.

47. Longwe H, Phiri KS, Mbeye NM, Gondwe T, Jambo KC, Mandala WL. Proportions of CD4+, CD8+ and B cell subsets are not affected by exposure to HIV or to Cotrimoxazole prophylaxis in Malawian HIV-uninfected but exposed children. BMC Immunol. 2015;16:50. 